\title{
Estimation of Genetic Parameters on Leg Score and Joint Cartilage Lesion Scores in a Closed Population of Duroc Pig
}

\author{
Kazuo FUKAWA, Toshie SUGIYAMA ${ }^{1}$, Seiji KUSUHARA ${ }^{1}$, Osamu KUDOH \\ and Kenji KAMEYAMA \\ Central Research Institute for Feed and Livestock, ZEN-NOH, Tsukuba-shi 300-4204, Japan \\ ${ }^{1}$ Faculty of Agriculture, Niigata University, Niigata-shi 950-2181, Japan
}

(Received November 11, 1999 ; Accepted April 21, 2000)

\begin{abstract}
Closed population of 6 generations Duroc pigs was selected for higher productivity and better leg soundness. The leg joint lesions of 174 slaughtered pigs at approximately $90 \mathrm{~kg}$ live weight were scored from 0 (no lesion) to 4 (very severe lesion) in optical, radiographical and histological standpoints throughout the six generations. Moreover, one thousand and three hundred eighty three pigs were scored from 1 (very good) to 5 (very weak) for leg weakness. Significant differences in the leg weakness score and joint lesion scores were observed among selected generations. Significant differences were observed between gilt and boar/barrow group in 9 traits. The heritabilities of joint lesion scores were low through intermediate. The heritabilities of histological joint scores were also low through intermediate. The phenotypic and genetic correlations between the scores of front (humerus and ulna) and rear legs (femur) were low. The genetic correlations between optical, radiographical and histological scores of the same joint were generally high. The phenotypic correlations between the leg weakness score and joint lesion scores were positive although statistically not significant from zero. The genetic correlations between the leg weakness score and joint lesion scores were generally intermediately or highly positive. The result suggested that selection based on joint lesion scores could lead to a better leg joint condition optically and histologically. It also suggested that selection based on leg weakness score could lead to the reduction of leg joint lesion according to the correlated response.
\end{abstract}

Animal Science Journal 71 (4) : 353-362, 2000

Key words : Duroc pig, REML, Osteochondrosis, Leg weakness

Leg weakness of pigs is one of the most serious problems in pig industry. Leg weakness leads to the reduction of the longevity of breeding pigs. It can also be said that according to the severity of leg weakness, premature culling rate of breeding animal increases, and the reduction of selection intensity could result in the loss of genetic improvement in economic traits. Consequently, leg weakness of pigs can cause large economic loss in pig industry ${ }^{10}$.

Although leg weakness in pigs has been investigated intensively by many authors, the exact cause remains uncertain $^{15)}$. In spite that osteochondrosis is not exactly the same as leg weakness, it was suggested that leg weakness is closely associated with osteochondrosis $^{8,9,15,17)}$. On the other hand, other researchers found no relationship between osteochondrosis and leg weakness, ${ }^{5,11}$. Lundeheim ${ }^{13)}$ has suggested that only when the joint lesion was severe, that there existed a significant relationship between osteochondrosis and leg weakness. Hill9) described that osteochondrosis was the syndrome causing degenerative joint disease or limb deformity in young, fast-growing pigs of both genders. Grøndalen ${ }^{7}$ described osteochondrosis as primary lesion that led to a failure of endochondral ossification in joint cartilage and growth plates, and suggested that osteochondrosis

Corresponding : Kazuo FUKAWA (fax : +81 (0) 298-69-1071, e-mail : fukawa@zk.zennoh.or.jp) 
FUKAWA, SUGIYAMA, KUSUHARA, KUDOH and KAMEYAMA

Table 1. Names and abbreviations of leg joint scores

\begin{tabular}{|c|c|c|c|}
\hline \multicolumn{2}{|c|}{ The name of leg distal joint scores } & \multirow{2}{*}{$\frac{\text { Abbreviation }}{\text { FMS }}$} & \multirow{2}{*}{$\frac{\text { Explanation }}{0: \text { No lesion to } 4: \text { very severe region }}$} \\
\hline Optical score & Femur & & \\
\hline & Humerus & HMS & $0:$ No lesion to $4:$ very severe region \\
\hline & Ulna & UMS & $0:$ No lesion to $4:$ very severe region \\
\hline & Total & TMS & "The total of FMS, HMS and UMS" \\
\hline \multirow[t]{4}{*}{ Radiographical score } & Femur & FXS & $0:$ No lesion to $4:$ very severe region \\
\hline & Humerus & HXS & $0:$ No lesion to $4:$ very severe region \\
\hline & Ulna & UXS & 0 : No lesion to 4 : very severe region \\
\hline & Total & TXS & "The total of FXS, HXS and UXS" \\
\hline \multirow[t]{11}{*}{ Histological score } & Cleft & ClHS & $\begin{array}{l}\text { "Total score of cleft in femur, humerus and } \\
\text { ulna" }\end{array}$ \\
\hline & Retain & RHS & $\begin{array}{l}\text { "Total score of retain in femur, humerus } \\
\text { and ulna" }\end{array}$ \\
\hline & Lysis & LHS & $\begin{array}{l}\text { "Total score of lysis in femur, humerus and } \\
\text { ulna" }\end{array}$ \\
\hline & Necrosis & NHS & $\begin{array}{l}\text { "Total score of necrosis in femur, humerus } \\
\text { and ulna" }\end{array}$ \\
\hline & Detachment & DHS & $\begin{array}{l}\text { "Total score of detachment in femur, } \\
\text { humerus and ulna" }\end{array}$ \\
\hline & Proliferation & PHS & $\begin{array}{l}\text { "Total score of proliferation in femur, } \\
\text { humerus and ulna" }\end{array}$ \\
\hline & Cluster & CuHS & $\begin{array}{l}\text { "Total score of cluster in femur, humerus } \\
\text { and ulna" }\end{array}$ \\
\hline & Femur & FHS & Total histological score of femur \\
\hline & Humerus & HHS & Total histological score of humerus \\
\hline & Ulna & UHS & Total histological score of ulna \\
\hline & Total & THS & "Total of FHS, HHS and UHS" \\
\hline Leg weakness score & & LWS & $1:$ very sound to $5:$ very weak \\
\hline
\end{tabular}

might be the same condition as that called osteochondritis in humans and dogs. In humans and dogs, osteochondritis gives rise to osteochondritis dissecants and finally leads to osteoarthrosis that is significantly associated with lameness.

It was shown that osteochondrosis was found in nearly $100 \%$ of growing young pigs ${ }^{7,13,16)}$. Grondalen $^{7}$ has shown that osteochondrosis was found in both growing pigs and young breeding pigs in

Anim. Sci. J. 71 (4) : 353-362, 2000 the same site of joints, and it was less common in pigs of more than one to one and half years of age. $\mathrm{He}$ suggested that after sexual maturity, the lesion that had not reached the surface of joint cartilage could completely heal or be repaired, and lesions that had reached the surface of joint cartilage gave rise to osteoarthrosis. Osteoarthrosis and proliferative osteitis could apparently give rise to lameness in pigs $^{1,7)}$. Histological and histochemical investigation 
Genetic Parameters of Leg and Joint Scores

Table 2. Basic statistics of joint lesion scores and leg weakness score

\begin{tabular}{llrrrrrrrr}
\hline \multicolumn{1}{c}{ Trait } & & No of data & Average & SD & min & max & skewness & \multicolumn{1}{c}{ kurtosis } \\
\hline Optical score & Femur & 322 & 0.112 & 0.379 & 0 & 3 & 3.922 & 17.723 \\
& Humerus & 322 & 0.422 & 0.891 & 0 & 3 & 2.064 & 2.943 \\
& Ulna & 322 & 0.845 & 1.000 & 0 & 3 & 0.897 & -0.390 \\
& Total & 322 & 1.379 & 1.449 & 0 & 7 & 1.101 & 0.989 \\
\hline \multirow{2}{*}{ Radiographical } & Femur & 322 & 0.596 & 0.796 & 0 & 4 & 1.370 & 1.652 \\
score & Humerus & 322 & 0.544 & 1.076 & 0 & 4 & 1.914 & 2.450 \\
& Ulna & 322 & 1.134 & 1.135 & 0 & 4 & 0.839 & -0.110 \\
& Total & 322 & 2.273 & 1.812 & 0 & 9 & 1.065 & 1.022 \\
\hline \multirow{2}{*}{ Histological score } & Cleft & 321 & 1.221 & 1.201 & 0 & 5 & 0.795 & 0.096 \\
& Retain & 321 & 1.206 & 1.395 & 0 & 7 & 1.305 & 1.477 \\
& Lysis & 321 & 2.006 & 1.714 & 0 & 7 & 0.770 & -0.067 \\
& Necrosis & 321 & 2.838 & 1.818 & 0 & 8 & 0.460 & -0.250 \\
& Detachment & 321 & 1.446 & 1.557 & 0 & 7 & 1.008 & 0.573 \\
& Proliferation & 321 & 3.343 & 1.921 & 0 & 9 & 0.257 & -0.157 \\
& Cluster & 321 & 2.408 & 1.624 & 0 & 8 & 0.536 & 0.086 \\
& Femur & 322 & 4.780 & 4.618 & 0 & 21 & 0.761 & 0.016 \\
& Humerus & 322 & 3.863 & 5.550 & 0 & 21 & 1.275 & 0.367 \\
& Ulna & 321 & 5.838 & 4.839 & 0 & 19 & 0.638 & -0.423 \\
& Total & 321 & 14.467 & 9.021 & 0 & 44 & 0.642 & -0.123 \\
\hline
\end{tabular}

of osteochondrosis has been done ${ }^{2,3)}$. However, the etiology and cause of osteochondrosis and leg weakness are still not understood.

The genetic relation between leg weakness and osteochondrosis was studied by many research$\mathrm{ers}^{12-14,19)}$. The heritabilities of osteochondrosis were similar to those of leg weakness ${ }^{13,14}$. The genetic correlation between leg weakness scores and leg joint scores was investigated by several researchers ${ }^{12-14)}$. Joint cartilages were usually scored by the observation of the surface of joint cartilage. No researchers have scored the osteochondrosis on histological level.

In this study, we investigated the effect of selection based on leg weakness score on the improvement of leg soundness and the reduction of leg joint lesion. We also estimated the genetic parameters of the leg weakness score and joint lesion scores that are necessary for the improvement of leg soundness and the reduction of leg joint lesion.

Anim. Sci. J. 71 (4) : 353-362, 2000

\section{Materials and Methods}

\section{Data}

The data set consists of lesion scores on 322 joint cartilages of 174 individual pigs. To measure the severity of osteochondrosis, pigs were scored leg joint lesions on optical, radiographical and histological standpoints. The number of pigs scored joint lesions in each generation ranged from 23 (generation 2 and 6) to 47 (generation 5). Most of the pigs have two repeated scores of left and right legs' joint cartilages. Additionally, leg weakness scores of 1,383 pigs were evaluated. The number of pigs scoring in each generation ranged from 169 (generation 2) to 323 (generation 6).

The breed of pigs is Duroc born from 1991 to 1997. These pigs were selected for their higher growth rate, larger eye muscle area, less back fat depth and better leg soundness in six generations. 
FUKAWA, SUGIYAMA, KUSUHARA, KUDOH and KAMEYAMA

Table 3. Least square means of each joint lesion

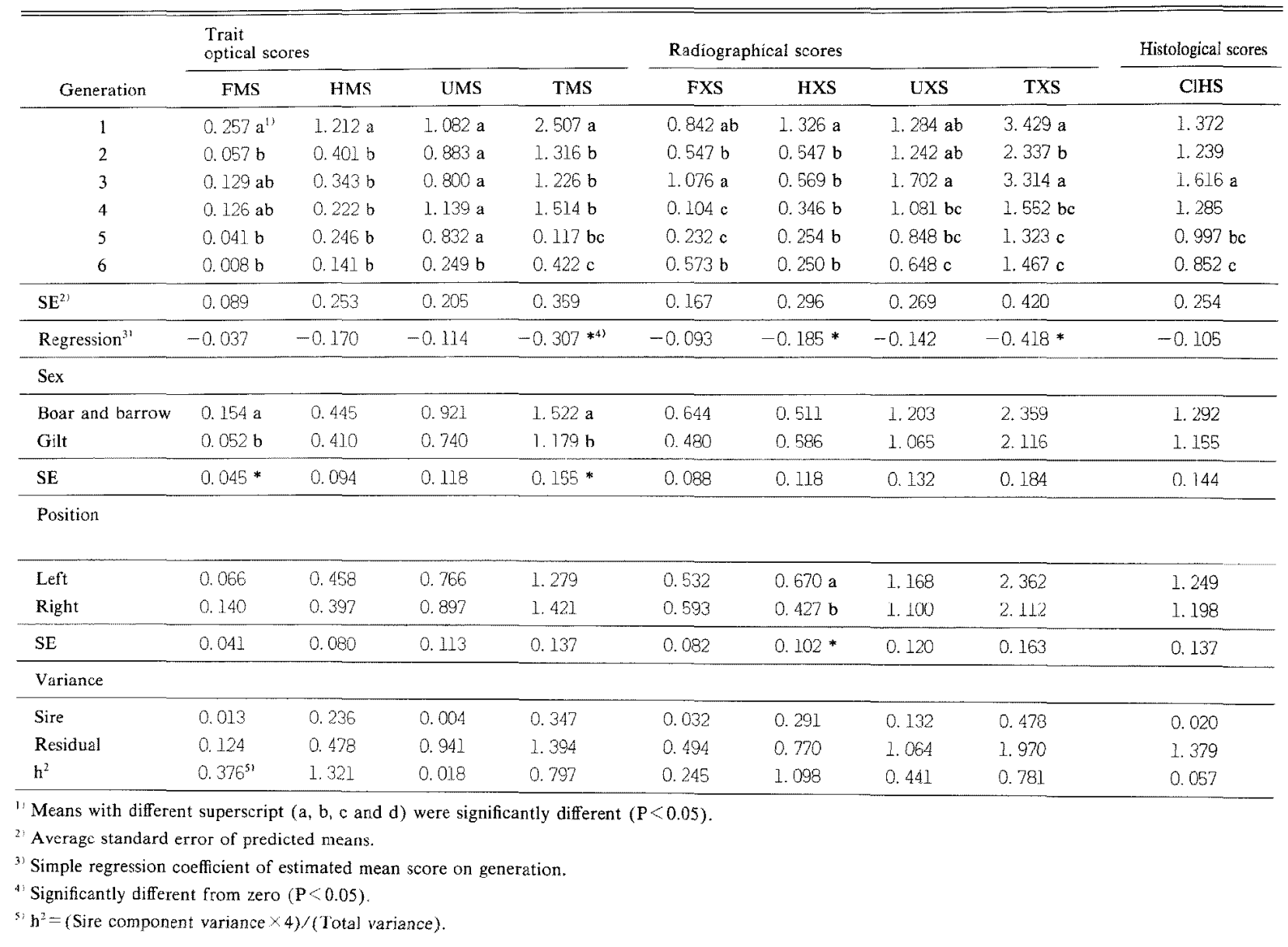

Pigs were reared in ad-libitum feeding regime from 30 to $90 \mathrm{~kg}$ live weight. Pigs were fed total digestible nutrient (TDN) $77 \%$, crude protein (CP) $12 \%$ feed from 30 to $60 \mathrm{~kg}$ live weight, and fed TDN 68 to $70 \%$, CP $12 \%$ feed from 60 to $90 \mathrm{~kg}$ live weight.

Pigs were reared in two different farms located in Ibaraki prefecture and Kumamoto prefecture. Pigs of third generation were farrowed by caesarian operation and fed milk substitute from day 0 to day 20 after birth. Pigs of other generations were farrowed and suckled in ordinary way.

The measured traits are indicated in Table 1. Pigs weighing approximately $90 \mathrm{~kg}$ were slaughtered and left and right bones (humerus, ulna and femur) were removed from carcasses. The distal joints of humerus and femur and distal epiphiseal cartilage of ulna were investigated optically and scored from 0 (no lesion) to 4 (severe lesion). After optical scoring, these joints were sliced at the width of $7 \mathrm{~mm}$ and radiographically investigated using soft $\mathrm{X}$-ray machines (Sofron, SRO-type $M$ and Softex MW100), and also scored from 0 (no lesion) to 4 (severe lesion). For histological investigation, the sliced bones were fixed with $10 \%$ formalin, and decalcified by aluminium chlorinate acute decalcification fluid.

The fixed bones were sliced at the width of $10 \mu \mathrm{m}$ using microtome, and were stained with haematoxylineosin or haematoxylin-fastgreen-safranin methods. The samples were scored on seven histological categories (cleft, retain, lysis, necrosis, detachment, proliferation and cluster). The seven scores of the three different joints were from 0 (no lesion) to 4 (severe lesion). These scores were summed up on each bone and on histological categories as shown in 
Genetic Parameters of Leg and Joint Scores

and leg weakness score

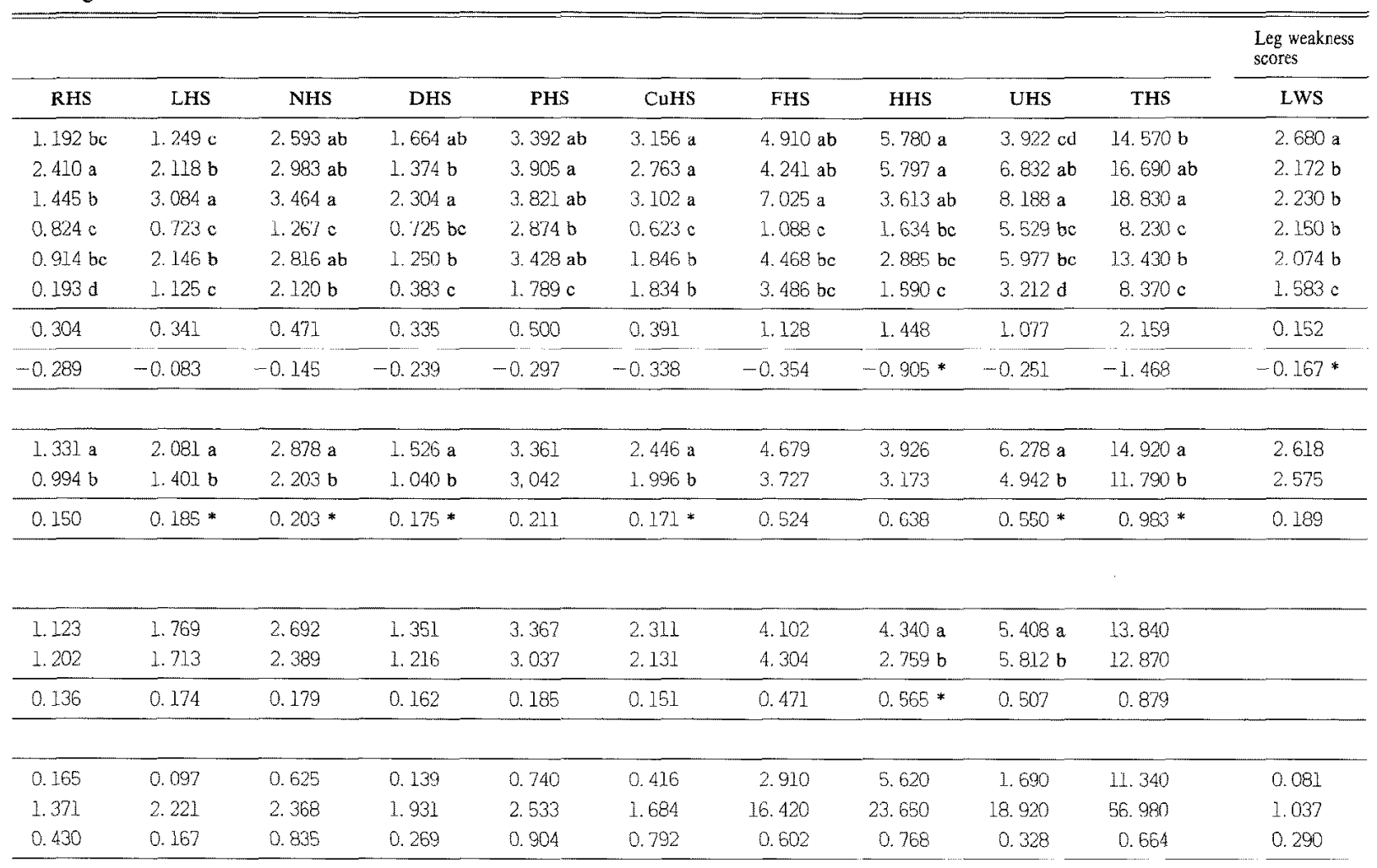

Table 1. Leg weakness scores were from 1 (very strong) to 5 (very weak) based on leg soundness of performance tested pigs at the weight of approximately $90 \mathrm{~kg}$.

\section{Statistical procedure}

For estimating the mean of fixed effects and variance components of sire, residual maximum likelihood (REML) procedure of GENSTAT ${ }^{4}$ was used. The statistical model for the analysis is

$$
\mathrm{Y}_{\mathrm{j} \mathrm{jkm}}=\mu+\mathrm{G}_{\mathrm{i}}+\mathrm{SE}_{\mathrm{j}}+\mathrm{PO}_{\mathrm{k}}+\mathrm{SR}_{\mathrm{l}}+\varepsilon_{\mathrm{ijklm}} \text { (Model 1) }
$$

where $Y_{\mathrm{ijklm}}$ is measurement of $\mathrm{ijklmth}$ bone. The fixed model terms are $: \mu$ the overall mean; $G_{i}$ the fixed effect of ith generation $(i=1,6) ; \mathrm{SE}_{j}$ the fixed effect of $j$ th $\operatorname{sex}(j=1$ for boar and barrow, $=2$ for gilt) ; and $\mathrm{PO}_{\mathrm{k}}$ the fixed effect of left and right position ( $k=1$ for right, 2 for left). The random model terms are : $\mathrm{SR}_{1}$ the random effect of Ith sire ; and $\varepsilon_{\mathrm{ijk} k \mathrm{~m}}$ the random residual error for observed data ijklm. Sire model was utilized in the estimation of both the mean of fixed effects and variance component of sire.

VCE3. $2^{6)}$ was used for estimating genetic parameters between the scored traits.

The statistical model for the analysis is

$$
\mathrm{Y}_{\mathrm{ijk \textrm {km }}}=\mu+\mathrm{G}_{\mathrm{i}}+\mathrm{SE}_{\mathrm{j}}+\mathrm{PO}_{\mathrm{k}}+\mathrm{AN}_{\mathrm{l}}+\varepsilon_{\mathrm{ijkm}} \text { (Model 2) }
$$

where fixed and random terms are the same as the previous model except that the random effect of sire is substituted by animal $\left(\mathrm{AN}_{1}\right)$.

For the estimation of genetic correlation, Model 2 is expanded to the multivariate linear mixed model. For genetic parameter estimation, individual animal model including pedigree information was utilized. 
FUKAWA, SUGIYAMA, KUSUHARA, KUDOH and KAMEYAMA

Table 4. Genetic parameters between joint lesion scores

\begin{tabular}{|c|c|c|c|c|c|c|c|c|c|c|}
\hline & FMS & HMS & UMS & TMS & FXS & $\mathrm{HXS}$ & UXS & TXS & ClHS & RHS \\
\hline FMS & $0.12(0.04)^{11}$ & $0.08(0.18)$ & $0.45(0.25)$ & $0.25(0.08$ & $-0.05(0.24)$ & $0.18(0.17)$ & $0.50(0.21)$ & $0.37(0.17)$ & $N E^{s\}}$ & $0.71(0.26)$ \\
\hline HMS & 0.13 & $0.45(0.05)$ & $0.09(0.14)$ & $0.80(0.06$ & $-0.19(0.14)$ & $0.84(0.04)$ & $0.07(0.11)$ & $0.50(0.09)$ & $0.00(0.19)$ & $0.53(0.11)$ \\
\hline TMS & $0,38 * * * 2=$ & $0.66^{* * *}$ & $0.72^{* * *}$ & $0.44(0.05$ & $-0.24(0.16)$ & $0.62(0.08)$ & $0.55(0.10)$ & $0.68(0.07)$ & $0.60(0.13)$ & $0.50(0.16)$ \\
\hline FXS & $0.34 * * *$ & 0.07 & 0.01 & 0.14 & $0.29(0.06)$ & $-0.22(0.13)-$ & $-0.14(0.14)$ & $0.29(0.13)$ & $0.57(0.22)$ & $0.08(0.20)$ \\
\hline HXS & 0.07 & $0.76^{* * *}$ & -0.01 & $0.48^{* * *}$ & 0.01 & $0.49(0.05)$ & $0.02(0.11)$ & $0.57(0.08)$ & $0.10(0.19)$ & $0.60(0.12)$ \\
\hline UXS & 0.09 & -0.02 & $0.61 * * *$ & $0.43^{* * *}$ & 0.13 & -0.02 & $0.42(0.05)$ & $0.62(0.07)$ & $0.57(0.13)$ & $0.27(0.18)$ \\
\hline TXS & $0.25 * * *$ & $0.47 * * *$ & $0.39 * * *$ & $0,62^{* * *}$ & $0.53 * *$ & $0.59 * * *$ & $0.67 * * *$ & $0.41(0.05)$ & $0.71(0.17)$ & $0.75(0.12)$ \\
\hline ClHS & 0.18 & 0.18 & 0.14 & $0.26^{* * *}$ & $0.24 * * *$ & 0.16 & $0.26 * * *$ & $0.37^{* * *}$ & $0.11(0.04)$ & $0.49(0.26)$ \\
\hline RHS & $0.24 * * *$ & $0.37 * * *$ & 0.07 & $0.34 * * *$ & $0.23 * * *$ & $0.43 * * *$ & 0.14 & $0.45 * * *$ & $0.34 * * *$ & $0.17(0.06)$ \\
\hline LHS & 0.13 & 0.16 & 0.03 & 0.15 & $0.24 * * *$ & $0.23^{* *}$ & 0.14 & $0.33^{* * *}$ & $0.38^{* * *}$ & $0.59 * * *$ \\
\hline NHS & 0.14 & $0.21 *$ & 0.07 & $0.22^{*}$ & $0.35 * * *$ & $0.32 * * *$ & 0.17 & $0.44 * * *$ & $0.37^{* * *}$ & $0.64^{* * *}$ \\
\hline DHS & 0.18 & $0,35 * * *$ & 0.06 & $0.31 * * *$ & $0.21 *$ & $0.44 * * *$ & 0.16 & $0.46^{* * * *}$ & $0.36 * *$ & $0.61 * * *$ \\
\hline CuHs & $0.21 *$ & $0.28 * * *$ & 0.07 & $0.28 * * *$ & $0.44 * * *$ & $0.34 * * *$ & $0.21^{*}$ & $0.52 * * *$ & $0.46^{* * *}$ & $0.57^{* * *}$ \\
\hline FHS & $0.36 * * *$ & 0.05 & 0.03 & 0.15 & $0.71^{* * *}$ & 0.00 & 0.12 & $0.39 * * *$ & $0.34 * * *$ & $0.38^{* * *}$ \\
\hline HHS & 0.02 & $0.58 * * *$ & -0.05 & $0.33^{* * *}$ & -0.03 & $0.72^{* * *}$ & -0.01 & $0.4 I^{* * * *}$ & $0.20 *$ & $0.59 * * *$ \\
\hline UHS & 0.06 & -0.08 & $0.29 * * *$ & 0.16 & 0.03 & -0.06 & $0.43^{* * *}$ & $0.25 * * *$ & $0.5 l^{* * *}$ & $0.41^{* * *}$ \\
\hline THS & $0.23 * *$ & $0.34 * * *$ & 0.14 & $0.36 * * *$ & $0.36^{* * *}$ & $0.41 * * *$ & $0.28 * * *$ & $0.58 * * *$ & $0.57 * *$ & $0.78 * * *$ \\
\hline LWS & -0.11 & 0.19 & 0.05 & 0] 4$. & -0.01 & 0.12 & 0.14 & 0.16 & 0.16 & 0.17 \\
\hline
\end{tabular}

Diagonal : heritability (in bold).

Upper triangle : Genetic correlation.

Lower triangle : Phenotypic correlation.

"In parenthesis : standard error of genetic correlation or heritability.

2)*** : Significantly different from zero $(\mathrm{P}<0.005)$.

${ }^{3) * *}$ : Significantly different from zero $(P<0.01)$.

4) : Significantly different from zero $(P<0.05)$.

${ }^{5}$ NE : Not estimable

\section{Results}

\section{Description of data}

The mean, standard deviation and other basic statistic values of each trait are shown in Table 2. For joint lesion scores, the optical, radiographical and histological scores of ulna tended to be consistently larger than those of femur or humerus. The score on each joint widely distributed over 4 or 5 classes of scores. Accordingly, all the traits were analyzed as linear traits rather than binomial traits.

\section{Comparison of fixed and random effects}

REML estimation of each fixed and random effect is shown in Table 3. In each generation, pigs that scored more than 3 in leg weakness score (weak and very weak) were excluded from the breeding population. A significant difference was found between generations in all the traits. In optical scores, the sixth generation had the smallest score of all generations. In radiographical scores, the sixth generation had the smallest scores in HXS and UXS, while the fourth generation had the smallest one in FXS and the fifth generation had the smallest one in TXS. Concerning histological score, the sixth generation had the smallest scores in RHS, DHS, PHS, HHS and UHS. On the other hand, the fourth generation had the smallest score in LHS, NHS, CuHS, FHS and THS. The sixth generation had significantly smaller scores than the first generation in most of scores except in FXS, LHS, NHS, FHS and UHS. The sixth generation also had the smallest score in LWS.

The simple linear regression coefficient of each score on generation was consistently negative. The coefficients of TMS, HXS, TXS, HHS and LWS were significantly different from zero $(P<0.05)$. There was a consistent tendency of reduction of each score as generation went on. As shown in Table 1, the smaller score stands for the better score. The tendency of improvement in joint cartilage condition and leg soundness was observed as the generation went on. 
Genetic Parameters of Leg and Joint Scores

and leg weakness score

\begin{tabular}{|c|c|c|c|c|c|c|c|c|c|}
\hline LHS & NHS & DHS & PHS & CuHs & FHS & HHS & UHS & THS & LWS \\
\hline $\mathrm{NE}$ & $0.32(0.20)$ & $0.77(0.26)$ & $-0.04(0.20)$ & $0.25(0.22)$ & $0.42(0.18)$ & $0.70(0.22)$ & $0.33(0.35)$ & $0.53(0.20)$ & $0.08(0.23)$ \\
\hline $0.33(0.44)$ & $0.13(0.13)$ & $0.44(0.15)$ & $0.40(0.11)$ & $0.23(0.12)$ & $-0.21(0.14)$ & $0.83(0.07)$ & $-0.03(0.17)$ & $0.37(0.11)$ & $0.31(0.14)$ \\
\hline $\mathrm{NE}$ & $0.55(0.19)$ & $0.11(0.26)$ & $0.75(0.16)$ & $0.78(0.26)$ & $0.14(0.16)$ & $0.15(0.20)$ & $0.48(0.23)$ & $0.59(0.10)$ & $0.75(0.25)$ \\
\hline $\mathrm{NE}$ & $0.40(0.12)$ & $0.39(0.17)$ & $0.59(0.10)$ & $0.57(0.15)$ & $0.02(0.14)$ & $0.78(0.13)$ & $0.19(0.18)$ & $0.58(0.11)$ & $0.33(0.16)$ \\
\hline $0.49(0.48)$ & $0.53(0.14)$ & $0.14(0.23)$ & $0.50(0.13)$ & $0.37(0.15)$ & $0.98(0.06)$ & $0.08(0.16)$ & $0.30(0.21)$ & $0.37(0.15)$ & $0.48(0.16)$ \\
\hline $0.60(0.40)$ & $0.29(0.12)$ & $0.67(0.12)$ & $0.47(0.12)$ & $0.22(0.12)$ & $-0.03(0.10)$ & $0.88(0.05)$ & $0.04(0.11)$ & $0.52(0.10)$ & $0.25(0.16)$ \\
\hline $0.10(0.44)$ & $0.30(0.14)$ & $0.01(0.20)$ & $0.55(0.11)$ & $0.29(0.13)$ & $0.17(0.13)$ & $-0.17(0.13)$ & $0.66(0.17)$ & $0.29(0.13)$ & $0.46(0.17)$ \\
\hline $0.69(0.40)$ & $0.79(0.20)$ & $0.62(0.13)$ & NE & $0.73(0.12)$ & $0.53(0.10)$ & $0.56(0.11)$ & $0.49(0.18)$ & $0.85(0.01)$ & $0.57(0.16)$ \\
\hline NE & $0.54(0.21)$ & $0.56(0.32)$ & $0.44(0.20)$ & $0.71(0.22)$ & $0.59(0.22)$ & $0.15(0.23)$ & $0.68(0.22)$ & $0.68(0.18)$ & $0.51(0.33)$ \\
\hline $0.52(0.51)$ & $0.61(0.14)$ & $0.64(0.17)$ & $0.89(0.10)$ & $0.78(0.12)$ & $-0.08(0.22)$ & NE & $0.42(0.14)$ & $0.77(0.09)$ & $0.76(0.20)$ \\
\hline $0.03(0.03)$ & $0.94(0.26)$ & $0.66(0.23)$ & $0.59(0.37)$ & NE & $0.67(0.47)$ & $0.78(0.62)$ & $0.48(0.56)$ & $\mathrm{NE}$ & NE \\
\hline $0.80^{* * *}$ & $0.27(0.05)$ & $0.40(0.19)$ & $0.76(0.09)$ & $0.86(0.06)$ & $0.69(0.10)$ & $0.43(0.14)$ & $0.18(0.19)$ & $0.83(0.05)$ & $0.24(0.17)$ \\
\hline $0.75^{* * *}$ & $0.69^{* * *}$ & $0.12(0.04)$ & $0.67(0.15)$ & $0.53(0.18)$ & $0.26(0.20)$ & $0.67(0.13)$ & $\mathrm{NE}$ & $0.76(0.11)$ & $0.59(0.25)$ \\
\hline $0.55^{* * *}$ & $0.63^{* * *}$ & $0.53^{* * *}$ & $0.34(0.06)$ & $0.96(0.06)$ & $0.42(0.13)$ & $0.64(0.12)$ & $0.73(0.22)$ & $0.91(0.01)$ & $0.81(0.14)$ \\
\hline $0.65^{* * *}$ & $0.77^{* * * m}$ & $0.58^{* * *}$ & $0.61 * * *$ & $0.28(0.06)$ & $0.59(0.12)$ & $0.56(0.14)$ & $0.46(0.18)$ & $0.99(0.04)$ & $0.43(0.16)$ \\
\hline $0.49 * * *$ & $0.60 * * *$ & $0.32 * * *$ & $0.48 * * *$ & $0.58^{* * *}$ & $0.28(0.05)$ & $0.13(0.16)$ & $0.06(0.21)$ & $0.60(0.11)$ & $0.38(0.16)$ \\
\hline $0.49^{* * *}$ & $0.52^{* * *}$ & $0.60^{* * *}$ & $0.51 * * *$ & $0.53^{* * *}$ & 0.01 & $0.29(0.06)$ & $0.10(0.21)$ & $0.71(0.09)$ & $0.50(0.21)$ \\
\hline $0.57^{* * *}$ & $0.50^{* * *}$ & $0.52^{* * *}$ & $0.45^{* * *}$ & $0.40^{* * *}$ & 0.11 & 0.01 & $0.14(0.06)$ & $0.44(0.17)$ & $-0.05(0.22)$ \\
\hline $0.86^{* * *}$ & $0.90^{* * *}$ & $0.81^{* * *}$ & $0.80^{* * *}$ & $0.84^{* * *}$ & $0.58^{* * *}$ & $0.62^{* * *}$ & $0.60^{* * *}$ & $0.29(0.06)$ & $0.49(0.17)$ \\
\hline 0.08 & 0.10 & 0.25 & 0.12 & 0.05 & -0.03 & 0.13 & 0.16 & 0.16 & $0.19(0.03)$ \\
\hline
\end{tabular}

Gilt had significant smaller scores $(P<0.05)$ than boars and barrows in FMS, TMS, ClHS, RHS, LHS, NHS, DHS, CuHS, UHS and THS. In the effect of the position (left and right), the difference was not significant in most of the traits except in HXS and HHS. Additionally, there was inconsistency in the difference between left and right positions.

From the estimated sire variance component, heritabilities ranged from 0.018 (UMS) to 1.321 (HMS).

\section{Genetic parameter estimation}

The estimated heritabilities, phenotypic and genetic correlations in the traits are shown in Table 4. The estimated heritabilities ranged from 0.03 (LHS) to 0.49 (HXS). The heritabilities obtained from animal model estimation (Table 4) were not consistent with those estimated from sire model (Table 3 ).

The heritabilities of humerus scores ranged between intermediate and high values ( 0.29 in HHS to 0.49 in HXS). Those of ulna ranged between low and high values ( 0.14 in UHS to 0.42 in UXS). Those of femur ranged between low to intermediate values ( 0.12 in FMS to 0.29 in FXS). The heritabilities of humerus and ulna tended to be higher than those of femur. The heritabilities of total scores were variable between intermediate and high values ( 0.29 in THS to 0.44 in TMS). The heritabilities of radiographical scores ( 0.29 in FXS to 0.49 in HXS) tended to be higher than those of optical scores (0.12 in FMS to 0.45 in HMS) and of histological ones (0.14 in UHS to 0.29 in HHS). In histological scores of the seven categories, the score of proliferation (PHS ; 0.34 ) had the highest heritability, while the score of lysis (LHS ; 0.03 ) had the lowest one. The heritability of leg weakness score was low (0.19).

In phenotypic correlation, the correlation between lesion scores on the different bone positions was low (from -0.02 in HXS-UXS to 0.13 in FXS-UXS). The correlations between optical, radiographical and histological scores of the same joint were from

Anim. Sci. J. 71 (4) : 353-362, 2000 


\section{FUKAWA, SUGIYAMA, KUSUHARA, KUDOH and KAMEYAMA}

intermediate to high values (from 0.29 in UMS-UHS to 0.76 in HMS-HXS). The correlations between aggregate scores were from intermediate to high values (from 0.36 in TMS-THS to 0.62 in TXS-THS). The correlations between leg weakness score (LWS) and joint scores of humerus and ulna were low and positive (from 0.05 in UMS-LWS and CuHs-LWS to 0.25 in DHS-LWS). However, the correlations between LWS and femur scores were slightly negative values and not significantly different from zero.

In genetic correlations, several correlations could not be estimated because REML program did not converged. Like phenotypic correlations, the correlations between the scores of different bones were generally low or negative value, although the correlation between FMS and UMS was high (from -0.22 in FXS-HXS to 0.13 in FHS-HHS, except 0.45 in FMSUMS).

The correlations between optical, radiographical and histological scores of the same position were highly positive (from 0.42 in FMS-FHS to 0.98 in FXS-FHS) except in FMS-FXS $(-0.05)$. Similar to the phenotypic correlations, genetic correlations between the seven categories' histological scores were from intermediate to high (from 0.40 in NHS-DHS to 0.96 in PHS-CuHS).

The correlations between the histological scores and aggregate joint lesion scores were generally high (from 0.39 in TMS-DHS to 0.99 in CuHS-TMS). Genetic correlations between the leg weakness score and joint lesion scores were generally from intermediate to high values except in FMS-LWS (0.08) and UHS-LWS $(-0.05)$. However, the correlation between the radiographical joint lesion score of humerus showing the highest heritability and the leg weakness score was not high $(0.25)$.

\section{Discussion}

In this study, pigs were selected for better leg soundness by independent culling. The result indicated that leg soundness was improved throughout six generations of selection. Additionally, joint lesion scores were reduced after six generations of selection.

In most of the traits, the positional difference was not significant. This may imply that left and right joint lesion scores could be regarded as the repeated scores of the same trait. The reason of inconsistency in heritabilities between animal model and sire model estimation may be that the pedigree information was included in the animal model, while the information were not included in the sire model. Since the pedigree information is included in animal model REML procedure, the estimated genetic parameters reflect both sib covariance and parent offspring covariance, while sire model estimation with no pedigree information only reflect half sib covariance. Therefore, the heritabilities of animal model estimation would be more reliable than those of sire model estimation.

The estimated heritabilities of joint lesion scores were generally consistent with those estimated by other researchers ${ }^{13,19)}$. Lundeheim ${ }^{13)}$ estimated heritabilities of low or intermediate values in joint and leg weakness scores. No researchers seem to have estimated heritabilities of histological lesion scores. Therefore, direct comparison to other results is impossible.

In this study, the estimated heritabilities of histological scores were similar to those of optical and radiographical scores, and the genetic correlations between optical, radiographical and histological scores in the same position were high. These scores may evaluate the same symptom, although the approach is different. This study suggested that those scores were intermediately heritable and that the improvement for better leg joint might be possible using the optical, radiographical or histological joint lesion scores. Since radiographical joint lesion scores generally had higher heritabilities than optical and histological joint lesion scores, radiographical observation of joint lesion may be more effective on selecting pigs for better leg joint condition using the information of slaughtered relatives. In individual selection, on the other hand, histological joint lesion score could be more useful because it could be measured from live pigs using a very small sample of cartilage obtained by biopsy method, although there may be the risk of infectious arthritis. Moreover, since there were high positive genetic relationship between the leg weakness score and joint lesion scores, multiple trait selection 
using these scores may be more effective than the single trait selection.

As for both genetic and phenotypic correlations between the lesion scores, the correlations between the joint lesion scores of the different bones were generally low in this research. Rothschild and Christian ${ }^{\text {i8) }}$ reported that the front leg joint cartilage had more severe lesion than the hind joint in Duroc pigs, which is consistent with our result. On the other hand, Lundeheim ${ }^{13)}$ reported that the genetic correlations between the elbow and knee joint scores were highly positive $(0.36$ in Landrace and 0.44 in Large White pigs) and that the knee joint lesion score was more severe than the elbow joint lesion score in both Landrace and Large White pigs. According to the difference of body conformation, the ratio of load to front and hind legs may differ between Duroc, Large White and Landrace pigs. This may explain the difference in the front and hind joint lesion and the inconsistency of the genetic correlation between the front and hind joint lesion scores in Duroc and the two breeds. However, since feeding regime and the confinement could also affect the joint lesion scores, further experiment using the same condition would be required to compare the joint lesions among different breeds.

Although the correlations among the three measurement scores of the same joint were generally high, the correlation between the optical and radiographical scores was very low in femur. The reason may be that most of the lesions were very small and those could not be found optically, or the lesion existed under the surface of the cartilage. Most of the correlations between the histological scores of the seven categories were highly positive. It could be that the histological symptoms were the typical disorders of osteochondrosis, and most of the symptoms were observed in the same lesion.

The phenotypic correlations between radiographical scores and histological scores of the same position were very high (from 0.43 in ulna to 0.72 in humerus). These results indicate that histological lesion may be directly related to optical lesion.

In this study, joint lesions were scored on the optical, radiographical and histological standpoint and their genetic parameters were estimated. The result showed that these traits were intermediately heritable, and there was positive genetic association between the joint lesion scores and the leg weakness score.

The selection differentials of joint lesion scores were uncertain since the scores of selected pigs were not available. The unselected control line was not available either. Therefore, there was no sufficient evidence demonstrating that the improvement of joint lesion scores was the correlated response of selection on LWS. However, there was a tendency of improvement in most of the scores. Moreover, in TMS, HXS, THS and HHS indicating significant regression coefficient on the generation, the genetic correlations with LWS were relatively high (from 0.25 to 0.50 ) and the heritabilities were also relatively high (from 0.29 to 0.49 ). It was suggested that the improvement of leg joint might due to the correlated response of independent culling on LWS, although some of the scores indicating relatively high heritabilities and genetic correlations with LWS did not show significant regression on the generation. High genetic association between LWS and joint lesion scores indicated that multiple trait selection for both leg soundness and better leg joint condition may be an effective method for reducing leg weakness problem in pigs. Multiple trait BLUP selection method could utilize the information of leg joint scores and leg weakness scores effectively by estimating the breeding values of these scores in live breeding pigs.

To measure joint lesion scores, one needs to slaughter the pigs to score the leg joints. The development for biopsy sampling methods of leg joint cartilage could realize the scoring of joint cartilage lesion of live replacement pigs, and selection toward better joint condition would become much faster. The utilization of biochemical markers for detecting joint disorder by assaying specific substances in serum or urine could detect leg disorder easily and could help select better pigs more efficiently.

\section{References}

1) Blowey RW. Proliferative osteosis of femoral greater trochanter and humeral medial epicondyle as a cause of lameness in sows. Veterinary Record, 131: 


\section{FUKAWA, SUGIYAMA, KUSUHARA, KUDOH and KAMEYAMA}

312-315. 1992.

2) Ekman S, Rodrigues-Martinez H. Ultrastructural localization of alkaline phosphatase activity in the normal and osteochondrotic joint cartilage of growing pigs. Acta Anatomica, $140: 26-33.1991$.

3) Ekman S, Heinegard D. Immunohistochemical localization in matrix proteins in the femoral joint cartilage of growing commercial pig. Veterinary Pathology, $29: 514-520.1992$.

4) Genstat 5 committee. Genstat 5 Release 3 Reference Manual. Clarendon Press. Oxford. 1993.

5) Goedegebuure SA, Rothschild MF, Christian LL, Ross RF. Severity of osteochondrosis in three genetic lines of Duroc swine divergently selected for frontleg weakness. Livestock Production Science, 19 : 487498. 1988.

6) Groeneveld E. REML VCE Ver. 3.2 User's Guide. 1996.

7) Grøndalen T. Osteochondrosis and arthrosis in pigs I. Incidence in animal up to $120 \mathrm{~kg}$ live weight. Acta Veterinaria Scandinavica, 15 : 1-25. 1974.

8) Grøndalen T. Leg weakness in pig I. Incidence and relationship to skeltal lesions, feed level, protein and mineral supply. Acta Veterinaria Scandinavica, 15 : 555-573. 1974.

9) Hill MA. Cause of degenerative joint disease (osteoarthrosis) and dyschondroplasia (osteochondrosis) in pigs. Journal of American Veterinary Medical Association, 197 : 107-115.1990.

10) Hill MA. Economic relevance, diagnosis and countermeasures for degenerative joint disease (ostcochondrosis) in pigs. Journal of American Veterinary Medical Association, 197 : 254-259. 1990.

11) Jorgensen B. Effect of different energy and protein levels on leg weakness and osteochondrosis in pigs. Livestock Production Science, 41 : 171-181. 1995.

12) Kadowaki $\mathbf{H}$, Suzuki $K$, Ogawa $Y$, Itoh $M$. Relationship of leg soundness trait to meat performance and body size in Duroc swine. Japanese Journal of Swine Science, $35: 107-113.1998$. (in Japanese)

13) Lundeheim N. Genetic analysis of osteochondrosis and leg weakness in the Swedish pig progeny testing scheme. Acta Agriculturae Scandinavica, $37: 159$ 173. 1987.

14) Lundeheim $N$, Rydhmer $L$. Genetic analysis of osteochondrosis and leg weakness in the Swedish Landrace pig population. Proceeding of the 4th World Congress on Genetics Applied to Livestock Production, $15: 493-495.1990$.

15) Nakano T, Brenman JJ, Aherne FX. Leg weakness and osteochondrosis in swine: A review. Canadian Journal of Animal Science, 67 : 883-901, 1987.

16) Reiland S. Pathology of so-called leg weakness in the pig. Acta Radiologica (supplement), $358: 23-44$. 1978.

17) Reiland S, Ordell N, Lundeheim N, Olsson SE. Heredity of osteochondrosis, body constitution and leg weakness in the pig. A correlation investigation using progeny testing. Acta Radiologica (supplement), 358 : 123-137. 1978.

18) Rothschild MF, Christian LL. Genetic control of front-leg weakness in Duroc swine I. Direct response to five generation of divergent selection. Livestock Production Science, $19: 459-471.1988$.

19) Wcbb AJ, Russel WC, Sales DI. Genetics of leg weakness in performance tested boars. Animal Production, 36: 117-130. 1983. 